\title{
AVALIAÇÃO DA SATISFAÇÃO DO ATENDIMENTO FISIOTERAPÊUTICO EM AMBIENTE HOSPITALAR
}

\author{
EVALUATION OF PHYSIOTHERAPEUTIC CARE SATISFACTION IN A HOSPITAL \\ ENVIRONMENT
}

\section{André Camargo ${ }^{a^{*}}$, Jamila Gabrielle Gonçalves ${ }^{b^{*}}$, Débora Melo Mazzo ${ }^{c^{*}}$, Juliana Carvalho \\ Schleder ${ }^{\mathrm{d}^{*}}$}

andrehcamargo@hotmail.comª , jamilagoncalves@hotmail.com ${ }^{\mathrm{b}}$, deboramazzo@uol.com.brc, juschleder@yahoo.com.br ${ }^{\mathrm{d}}$ Hospital Universitário Regional dos Campos Gerais*

\section{RESUMO}

Esta pesquisa teve por objetivo avaliar o grau de satisfação em relação ao atendimento fisioterapêutico dos pacientes hospitalizados nas enfermarias do Hospital Universitário Regional dos Campos Gerais (HURCG). Participaram da pesquisa 55 pacientes, os quais responderam a um questionário brasileiro de satisfação validado para pacientes ambulatoriais, sendo este adaptado para o perfil da pesquisa. As questões versavam sobre: interação fisioterapeuta-paciente, estrutura física e satisfação geral. A hipótese inicial de encontrar nos dados boa satisfação dos pacientes com atendimento fisioterapêutico prestado, pôde ser comprovada na pergunta referente ao nível de satisfação geral, na qual demonstrou que 90,90\% (n=50) apontaram essa questão como "excelente" ou "ótima". O resultado obtido, de modo geral, foi satisfatório, porém, ressalta-se a necessidade da otimização da assistência fisioterapêutica através de capacitações sistemáticas de toda a equipe para a manutenção da qualidade técnica do atendimento, sempre incentivando a humanização e a importância da interação fisioterapeuta-paciente.

Palavras-chave: Fisioterapia; Serviço Hospitalar De Fisioterapia; Satisfação Do Paciente; Hospitais Universitários; Saúde Pública.

\section{ABSTRACT}

This research aimed to evaluate satisfaction degree regarding physiotherapeutic care of hospitalized patients in Hospital Universitário Regional dos Campos Gerais (HURCG). A total of 55 patients participated in the study, which answered a validated brazilian questionnaire for outpacients, which was adapted to the research profile. Issues concerned to: therapist-patient interaction, physical structure and overall satisfaction. Initial hypothesis of finding a good patient satisfaction with physiotherapeutic care provided was proven by the issuerelated to the level of overall satisfaction, which showed that $90.90 \%(n=50)$ considered this issue as "excellent" or "optimal". In a general way, theresult obtained was satisfactory however, it is necessary to optimize physical therapy assistance through systematic training of the entire team to maintain the technical quality of care, always encouraging humanization and the importance of physiotherapist-patient relationship.

Keywords: Physical Therapy Specialty; Physical Therapy Department, Hospital; Patient Satisfaction; Hospitals, University; Public Health. 


\section{Introdução}

O processo de reestruturação dos serviços públicos de saúde no Brasil, iniciado com a implantação do Sistema Único de Saúde (SUS) em 1988, trouxe consigo a necessidade de se repensar a respeito do desempenho desses serviços visando buscar a melhoria na qualidade, a qual se associa às características desejáveis na prestação do cuidado, tais como efetividade, eficiência, equidade, aceitabilidade, acessibilidade e adequabilidade ${ }^{1}$.

A qualidade dos serviços de saúde possui três aspectos: estrutura, processo e resultados. Estrutura refere-se aos recursos físicos, materiais e humanos existentes no âmbito da assistência à saúde; o processo abrange $\mathrm{o}$ atendimento direto empregado ao usuário/paciente; os resultados evidenciam os efeitos das ações e dos procedimentos sobre o estado de saúde após receber a assistência ${ }^{2}$. Ao investigara qualidade do atendimento, pode-se obter dados sobre a perspectiva do usuário, possibilitando encontrar soluções para otimizar os serviços prestados ${ }^{3}$.

A satisfação da população usuária do serviço de saúde pública vem sendo cada vez mais enfocada, visando à melhoria na qualidade deste serviço. Portanto, para mensurar a condição de saúde do paciente ou os resultados do tratamento realizado, são amplamente utilizados questionários e outros instrumentos ${ }^{1-10}$, já que a existência de um atendimento em saúde adequada pode ser almejada avaliando a qualidade do serviço prestado, buscando encontrar possíveis falhas ${ }^{4,5}$. No âmbito hospitalar, o serviço de saúde é constituído por uma equipe interdisciplinar da qual a fisioterapia é integrante, cujo objetivo é prevenir complicações e/ou restaurar as funções perdidas no internamento.

$\mathrm{O}$ atendimento fisioterapêutico costuma levar mais tempo do que uma consulta médica, as condutas envolvem maior contato físico e em geral requerem a participação ativa do paciente, além do mais, a terapia é capaz de provocar dor e como o paciente encontra-se em uma situação de vulnerabilidade, esta pode ser até mesmo encarada como uma ameaça física, podendo influenciar diretamente em sua satisfação durante o internamento ${ }^{1,6,7,8}$.
Nesse contexto, a qualidade do atendimento com o paciente vem sendo focada na avaliação do conhecimento pessoal, das habilidades e da experiência do profissional da saúde que realiza os cuidados diretos, já que a satisfação está ligada de forma sinérgica no que se refere ao sucesso do tratamento ${ }^{3}$. O paciente cria expectativas que devem serlevadas em conta por serem consideradas um dos maiores determinantes da satisfação; quando pacientes estão insatisfeitos, apresentam menor adesão ao tratamento, e consequente aumento no período de internamento ${ }^{1,8}$.

$\mathrm{Na}$ busca pela excelência na qualidade do atendimento com o paciente hospitalizado, o feedback sobre os atendimentos fisioterapêuticos realizados pode ser utilizado sistematicamente para melhorar os métodos de cuidados de saúde hospitalar ${ }^{11}$. Esta pesquisa teve por objetivo avaliar o grau de satisfação com o atendimento fisioterapêutico dos pacientes hospitalizados nas enfermarias do Hospital Universitário Regional dos Campos Gerais (HURCG).

\section{Metodologia}

Este estudo prospectivo, transversal, descritivo e analítico foi aprovado pelo Comitê de Ética em Pesquisa (CEP) da Universidade Estadual de Ponta Grossa (UEPG) sob o número de registro 1.990.534. Foram incluídos 57 pacientes adultos internados nas enfermarias do HURCG, dos quais dois foram excluídos por não responderem ao questionário na íntegra, ficando a amostra constituída por 55 pacientes. A coleta dos dados ocorreu no período de dois meses após a aprovação do CEP, no Hospital Universitário Regional dos Campos Gerais (HURCG).

Era requisito que o paciente possuísse nível cognitivo adequado para interpretar as questões e tivesse recebido ao menos cinco atendimentos fisioterapêuticos. Foi entregue o termo de consentimento livre e esclarecido a todos os pacientes, e após assiná-lo, foi apresentado o questionário de satisfação de atendimento fisioterapêutico adaptado da validação de Mendonça e Guerra ${ }^{12}$ o qual ficou disponível pelo período de $24 \mathrm{~h}$ para ser respondido. Esse 
questionário continha duas partes: a primeira preenchida pelo pesquisador que caracterizava o perfil do participante (sexo, idade, setor de internamento, nível de consciência, diagnóstico clínico e pneumo-funcional, grau de escolaridade, histório de tratamento fisioterapêutico e grau de entendimento quanto a prescrição médica para o tratamento fisioterapêutico), e a segunda parte com 22 questões que avaliavam a satisfação do paciente em relação a interação terapeutapaciente, estrutura física onde é realizado o atendimento fisioterapêutico e satisfação geral do atendimento fisioterapêutico.

\section{Resultados}

Mais da metade dos entrevistados (56,36\%, $\mathrm{n}=31$ ) precisaram de auxílio para responder ao questionário por motivos como: não conseguir escrever no momento, dificuldade de interpretação, ou por não serem alfabetizados, necessitando assim, ajuda de seu acompanhante. Os outros 43,63\%, ( $\mathrm{n}=24)$ não necessitaram de auxílio.

O sexo masculino correspondeu a $61,80 \%$ $(\mathrm{n}=34)$ dos entrevistados e o feminino a $38,2 \%$ $(\mathrm{n}=21)$. A mediana da idade foi de 52 , com primeiro quartil 32 anos e terceiro quartil 37 anos de idade.

As características da amostra estão descritas na Tabela 1. Como pode ser observado, os pacientes apresentavam baixo grau de escolaridade já que $87,27 \%(\mathrm{n}=48)$ não concluíram ensino médio.

Tabela 1 - Caracterização da amostra

\begin{tabular}{lcc}
\hline & N & \% \\
\hline Grau de escolaridade & 5 & 9,09 \\
Nenhuma & 16 & 29,09 \\
Fundamental incompleto & 18 & 32,72 \\
Fundamental completo & 9 & 16,36 \\
Ensino médio incompleto & 5 & 9,09 \\
Ensino médio completo & 2 & 3,63 \\
Ensino superior incompleto & & \\
Submetido a tratamento fisioterapêutico prévio & 41 & 74,54 \\
Nunca & 14 & 25,45 \\
Sim & & \\
Procedência & 43 & 78,18 \\
Unidade de terapia intensiva ou PA do HURCG & 12 & 21,81 \\
Direto (cirurgias eletivas) & & \\
Unidade de internamento & 19 & 34,54 \\
Cirurgia geral & 10 & 18,18 \\
Ortopedia & 9 & 16,36 \\
Neurologia & 5 & 9,09 \\
Clínica médica (complicações pulmonares) & 12 & 21,81 \\
Clínica médica (outras patologias) & & \\
Conhecimento do diagnóstico clínico & 50 & 90,90 \\
Sim & 5 & 9,09 \\
Não & 5 &
\end{tabular}




\section{Diagnóstico fisioterapêutico}

Alteração ventilatória

$22 \quad 40$

Alteração circulatória

$16 \quad 29,09$

Disfunções osteomioarticulares

$36 \quad 65,45$

Alterações neurocinéticofuncionais

$9 \quad 16,36$

Conhecimento do motivo da realização da fisioterapia

Sim

A Tabela 2 sintetiza as respostas relacionadas à interação fisioterapeuta-paciente, em que, $48,40 \%$ apontaram como excelente, $35,11 \%$ como ótimo, $14,89 \%$ como bom e apenas $1,60 \%$ como ruim.

Tabela 2 - Respostas referentes à interação fisioterapeuta-paciente

\begin{tabular}{|c|c|c|}
\hline & $\mathbf{N}$ & $\%$ \\
\hline \multicolumn{3}{|c|}{ Explicações dadas para realizar os exercícios } \\
\hline Excelente & 15 & 27,27 \\
\hline Ótimo & 25 & 45,45 \\
\hline Bom & 13 & 23,64 \\
\hline Ruim & 2 & 3,64 \\
\hline \multicolumn{3}{|c|}{ Técnicas e procedimentos aplicados de forma confortável } \\
\hline Excelente & 42 & 76,36 \\
\hline Ótimo & 10 & 18,18 \\
\hline Bom & 2 & 3,64 \\
\hline Ruim & 1 & 1,82 \\
\hline \multicolumn{3}{|c|}{ Linguagem de fácil compreensão usada } \\
\hline Excelente & 38 & 69,09 \\
\hline Ótimo & 13 & 23,64 \\
\hline Bom & 3 & 5,45 \\
\hline Ruim & 1 & 1,82 \\
\hline \multicolumn{3}{|c|}{ Aprofundamento na avaliação do seu problema } \\
\hline Excelente & 10 & 18,18 \\
\hline Ótimo & 32 & 58,18 \\
\hline Bom & 13 & 23,64 \\
\hline Ruim & 0 & 0 \\
\hline \multicolumn{3}{|c|}{ Habilidade do fisioterapeuta durante 0 atendimento } \\
\hline Excelente & 38 & 69,09 \\
\hline Ótimo & 15 & 27,27 \\
\hline Bom & 2 & 3,64 \\
\hline Ruim & 0 & 0 \\
\hline
\end{tabular}


Oportunidade dada para expressar sua opinião

Excelente 40

Ótimo

45,45

Bom

Ruim

$6 \quad 10,91$

$2 \quad 3,64$

Atenção dada às suas queixas

$\begin{array}{lcc}\text { Excelente } & 22 & 40 \\ \text { Ótimo } & 23 & 41,82 \\ \text { Bom } & 9 & 16,36 \\ \text { Ruim } & 1 & 1,82\end{array}$

Confiança nas orientações dadas pelo fisioterapeuta

Excelente

$27 \quad 49,09$

Ótimo

$25 \quad 45,45$

Bom

$3 \quad 5,45$

Ruim

0

0

Esclarecimento de suas dúvidas pelo fisioterapeuta

Excelente

$17 \quad 30,91$

Ótimo

Bom

1120

Ruim

$4 \quad 7,27$

Privacidada respeitada durante seu atendimento

Excelente

$35 \quad 63,64$

Ótimo

Bom

$5 \quad 9,09$

Ruim

Gentileza do fisioterapeuta

Excelente
Ótimo
Bom
Ruim

$40 \quad 72,73$

$10 \quad 18,18$

$4 \quad 7,27$

Ruim

$1 \quad 1,82$

Respeito e interesse com que você é tratado

$\begin{array}{lcc}\text { Excelente } & 26 & 47,27 \\ \text { Ótimo } & 21 & 38,19 \\ \text { Bom } & 8 & 14,54 \\ \text { Ruim } & 0 & 0\end{array}$

Segurança transmitida pelo fisioterapeuta

$\begin{array}{lcc}\text { Excelente } & 26 & 47,27 \\ \text { Ótimo } & 20 & 36,37 \\ \text { Bom } & 9 & 16,36 \\ \text { Ruim } & 0 & 0\end{array}$




\section{Explicações oferecidas com clareza}

Excelente

$28 \quad 50,90$

Ótimo

$13 \quad 23,64$

Bom

13

23,64

Ruim

1

1,82

A maioria dos pacientes classificaram a fisioterapêutico no HURCG como bom ou estrutura física onde foi realizado o tratamento excelente (Tabela 3).

Tabela 3 - Respostas referentes à estrutura física onde é realizada a fisioterapia

\begin{tabular}{lcc}
\hline & $\mathbf{N}$ & $\mathbf{\%}$ \\
\hline Condições gerais do ambiente hospitalar & 27 & 49,09 \\
Excelente & 18 & 32,73 \\
Ótimo & 10 & 18,18 \\
Bom & 0 & 0 \\
Ruim & & \\
Conforto do ambiente onde é realizada a fisioterapia & 14 & 25,45 \\
Excelente & 27 & 49,09 \\
Ótimo & 13 & 23,64 \\
Bom & 1 & 1,82 \\
Ruim & 1 & \\
Limpeza, higiene e segurança dos equipamentos/materiais utilizados pelo fisioterapeuta & 38,18 \\
Excelente & 21 & 43,64 \\
Ótimo & 24 & 16,36 \\
Bom & 9 & 1,82 \\
Ruim & 1 & \\
\hline
\end{tabular}

O mesmo foi observado quanto à satisfação geral dos pacientes com a fisioterapia (Tabela 4).

Tabela 4 - Respostas referentes à satisfação geral do atendimento fisioterapêutico

\begin{tabular}{lcc}
\hline & $\mathbf{N}$ & $\mathbf{\%}$ \\
\hline Serviço de fisioterapia no geral & & \\
Excelente & 41 & 74,54 \\
Ótimo & 9 & 16,36 \\
Bom & 4 & 7,28 \\
Ruim & 1 & 1,82 \\
Importância do fisioterapeuta na sua recuperação & & \\
Excelente & 39 & 70,91 \\
Ótimo & 12 & 21,82 \\
Bom & 3 & 5,45 \\
Ruim & 1 & 1,82
\end{tabular}




\section{Horário conveniente para o atendimento}

\begin{tabular}{lcc} 
Excelente & 18 & 32,73 \\
Ótimo & 26 & 47,27 \\
Bom & 11 & 20 \\
Ruim & 0 & 0 \\
Número de atendimentos fisioterapêuticos & & 30,91 \\
Excelente & 17 & 50,91 \\
Ótimo & 28 & 18,18 \\
Bom & 10 & 0 \\
Ruim & 0 & 0 \\
\hline
\end{tabular}

A Tabela 5 expõe ascorrelações de idade e escolaridade com as variáveis estudadas.

Tabela 5 - Verificação de correlação entre as variáveis estudadas

\begin{tabular}{l|c|c}
\hline Variáveis cruzadas & Valor de $\mathrm{r}$ & Valor $\mathrm{p}$ \\
\hline Idade x $1^{\mathrm{a}}$ vez que realizou fisioterapia & 0,207 & 0,130 \\
Idade x $1^{\mathrm{a}}$ vez internado no HURCG & 0,076 & 0,583 \\
Idade x sabe diagnóstico clínico & 0,291 & 0,031 \\
Idade x sabe motivo da fisioterapia & 0,182 & 0,182 \\
Escolaridade x sabe diagnóstico clínico & 0,089 & 0,893 \\
Escolaridade x sabe motivo da fisioterapia & 0,062 & 0,325 \\
\hline
\end{tabular}

\section{Discussão}

A presente pesquisa avaliou o grau de satisfação dos pacientes hospitalizados com o atendimento fisioterapêutico nas enfermarias do HURCG, visando à melhoria na qualidade do serviço de fisioterapia prestado.

Vanti et al. ${ }^{8}$ observaram, numa pesquisa para validação de um questionário de satisfação para pacientes hospitalizados, que a produção desse tipo de trabalho científico raramente é relatada. Relatam que as possíveis razões para isso podem ser: (a) o estado grave de saúde dos pacientes, tornando difícil administrar um questionário;(b) dificuldade em identificar com precisão quem são fisioterapeutas entre os profissionais da assistência; (c) quais entre os tratamentos recebidos são intervenções da fisioterapia; e até mesmo (d) o receio em registrar a atual situação do serviço público de saúde.

O HURCG é um hospital público com atendimento exclusivo pelo SUS; no presente estudo, a análise dos dados evidenciou baixo grau de escolaridade dos pacientes. Fréz e Nobre ${ }^{4}$, também num estudo de satisfação do serviço de fisioterapia em rede pública no Brasil, evidenciaram que $50,50 \%$ do total de 99 participantes teriam completado o ensino médio, entretanto, foi uma pesquisa realizada a nível ambulatorial. Corroborando com esses dados, o relatório final da Comissão Nacional sobre Determinantes Sociais da Saúde (CNDSS) de 2008, demonstrou que há predominância da população com menor renda e escolaridade dentre usuários do SUS ${ }^{13}$.

Gonçalves et al. citam em seu estudo uma pesquisa elaborada pelo Ministério da Saúde junto ao Conselho Nacional de Secretários da Saúde em 2003, que mostra que o SUS é utilizado por mais de 90\% dos brasileiros e que este dado é satisfatório para admitir que há baixa qualidade nos serviços oferecidos pela rede pública, tanto em relação aos equipamentos quanto às equipes profissionais ${ }^{14}$. Já os resultados da presente pesquisa discordam dessa afirmativa, pois somente $1,82 \%$ dos entrevistados classificaram a estrutura como ruim e quanto ao aspecto profissional, todos os quesitos apontados foram positivos, evidenciando uma boa qualidade do serviço prestado. 
Embora o HURCG não possua um ambiente específico para realização da fisioterapia, e, portanto, os atendimentos fisioterapêuticos sejam realizados no próprio quarto do paciente e quando necessário, levados a corredores, escadas e áreas externas, $49,09 \%(n=27)$ dos entrevistados classificaram a estrutura física onde são realizados os atendimentos como excelente e o restante como boa ou ótima. Boa satisfação nesse quesito foi encontrada numa pesquisa realizada por Brito et al. ${ }^{15}$ a nível ambulatorial em clínicas públicas e conveniadas ao SUS, na qual $87,20 \%(n=137)$ mostraram-se satisfeitos com o conforto do ambiente. Ainda nessa mesma pesquisa, 22,90\% $(\mathrm{n}=36)$ desses pacientes relataram não terem recebido informações sobre seu estado de saúde, já no presente estudo apenas $9,09 \%(n=5)$ não sabiam qual era o seu diagnóstico clínico.

Nas enfermarias do HURCG, os pacientes internados podem facilmente identificar o profissional fisioterapeuta, devido à identificação no jaleco/crachá, mostrando o nome e qualificação, e também por ser hábito dos profissionais se apresentarem ao paciente, informá-lo sob sua função e condutas a serem realizadas. Este quesito (interação fisioterapeuta-paciente) apontou satisfação positiva (Tabela 2), diferentemente do que foi encontrado no estudo de Vanti et al. ${ }^{8}$,

As correlações estatísticas (Tabela 5) não apresentaram evidências significativas, porém neste estudo, esse resultado pode ser um bom preditor, pois independentemente da idade, do nível de escolaridade, de saber seu diagnóstico ou o motivo de realizar fisioterapia, o atendimento dos fisioterapeutas foi considerado satisfatório, mostrando que estes estão capacitados a atenderem uma diversa gama de pacientes. Esse fato pode ser confirmado observando os resultados descritos na Tabela 2,em que: 'Explicações dadas para realizar o exercício', 'Linguagem de fácil compreensão usada', e 'Explicações oferecidas com clareza' foram apontados como 'excelente' pelos entrevistados ${ }^{14}$. Para Mendonça e Guerra ${ }^{12}$ a satisfação do paciente está intimamente ligada às habilidades comunicativas e afetivas do fisioterapeuta, sugerindo que o esclarecimento de dúvidas, a segurança e o respeito são fatores decisivos para uma experiência negativa ou positiva do paciente.

Somente o cruzamento entre a idade e saber o diagnóstico clínico obteve significância estatística $(\mathrm{p}=0,031)$, sendo que quanto mais jovens os pacientes, mais ciência tinham sobre seu diagnóstico, porém o grau de correlação é fraco $(\mathrm{r}=0,291)$. É importante ressaltar que apesar de 47,27\% ( $n=26)$ dos entrevistados não saberem o motivo pelo qual estavam realizando a fisioterapia, todos relataram que a atuação do fisioterapeuta é importante em sua recuperação (Tabela 4).Os pacientes, de modo geral, podem não estar aptos para avaliar os cuidados quanto às questões técnicas e à capacidade dos profissionais, porém, quando lhes são oferecidas oportunidades, podem expressar sua opinião quanto à interação com a equipe de saúde e a influência de suas ações ${ }^{16}$.

A hipótese inicial de encontrar nos dados boa satisfação dos pacientes com atendimento fisioterapêutico prestado, pôde ser comprovada na pergunta referente ao nível de satisfação geral, a qual demonstrou que $90,90 \%(n=50)$ apontaram essa questão como excelente ou ótima (Tabela 4).

O principal intuito dessa pesquisa foi de obter um feedback dos pacientes quanto ao tratamento proporcionado pelos fisioterapeutas do HURCG. Vale ressaltar que o número reduzido de pesquisas do mesmo aspecto dificultou comparação entre resultados. Detectamos também, dificuldade em conseguir responder ao questionário, por grande parte dos pacientes, devido às limitações físicas, sendo necessário auxílio de um terceiro para ler e/ou assinalar, podendo ter influenciado nas respostas. Outro ponto importante é que era necessário que o paciente estivesse lúcido e orientado, por este fato, optamos avaliar o grau de satisfação, em relação aos serviços prestados pela fisioterapia, apenas nas enfermarias e não no pronto atendimento e nas unidades de terapia intensiva, representando somente uma parcela dos serviços realizados pelos fisioterapeutas no HURCG. Supõe-se também, que o fato de o paciente responder ao questionário ainda durante seu internamento possa ter inibido respostas negativas.

O estudo levantou interesse dos supervisores do serviço de fisioterapia e da direção do HURCG por ser uma avaliação nunca realizada e também demonstrou sua importância, devido a um número reduzido de estudos na literatura científica referentes à avaliação da satisfação do atendimento fisioterapêutico de pacientes hospitalizados. 
Embora o resultado obtido, de modo geral, tenha sido satisfatório, ressalta-se a necessidade da otimização da assistência fisioterapêutica através de capacitações sistemáticas de toda a equipe para a manutenção da qualidade técnica do atendimento, sempre incentivando a humanização do atendimento e a importância da interação fisioterapeuta-paciente, além de avaliação periódica da satisfação dos pacientes, para que seja possível identificar e sanar possíveis falhas ao longo do tempo.

\section{Conclusão}

$\mathrm{O}$ atendimento fisioterapêutico dos pacientes hospitalizados no HURCG é, de forma geral, considerado satisfatório pelos usuários. $\mathrm{O}$ estudo aponta fatores que podem ser melhorados, com isso, medidas deverão ser tomadas para um atendimento de excelência.

\section{Referências}

1. Moreira CF, Borba JAM, Mendonça KMPP. Instrumento para aferir a satisfação do paciente com a assistência fisioterapêutica na rede pública de saúde. Fisio e Pesq. 2007;14(3):37-43.

2. Cavalheiro LV, Eid RAC, Talerman C, et al. Design of an instrument to measure the quality of care in Physical Therapy. Einstein. 2015;13(2):260-8.DOI: 10.1590/ S1679-45082015GS3248.

3. Rodrigues RM, Raimundo CB, Silva CK. Satisfação dos usuários dos serviços privados de fisioterapia do município de Campos dos Goytacares - RJ. Perspectivas Online. [www.perspectivasonline.com.br].2010;4(14).

4. Fréz AR, Nobre MIRS. Satisfação dos usuários dos serviços ambulatoriais de fisioterapia da rede pública. Fisioter. Mov.2011;24(3):419-428.

5. Machado NP, Nogueira LT. Avaliação da satisfação dos usuários de serviços de Fisioterapia. Rev Bras Fisioter. São Carlos. 2008;12(5):401-8.

6. Calthorpe S, Barber EA, Holland AE, et al. An intensive physiotherapy program improves mobility for trauma patients. J Trauma Acute Care Surg. 2014;76(1):101-106. DOI: 10.1097/TA.0b013e3182ab07c5.
7. Suda EY, Uemura MD, Velasco E. Avaliação da satisfação dos pacientes atendidos em uma clínicaescola de Fisioterapia de Santo André, SP. Fisio e Pesq. 2009;16(2):126-31.

8. Vanti C, Pillastrini P, Monticone M, et al. The Italian version of the Physical Therapy Patient Satisfaction Questionnaire - [PTPSQ-I(15)]: psychometric properties in a sample of inpatients. BMC Musculoskeletal Disorders. 2014,15:135.doi: 10.1186/1471-2474-15-135.

9. Vanti C, Monticone M, Ceron D, et al. Italian Version of the Physical Therapy Patient Satisfaction Questionnaire: Cross-Cultural Adaptation and Psychometric Properties. Phys Ther. 2013;93(7):911-922. DOI: 10.2522/ ptj.20120170.

10. Mendes ACG, de Araújo Jr JLCA, Furtado BMAS, et al. Avaliação da satisfação dos usuários com a qualidade do atendimento nas grandes emergências do Recife, Pernambuco, Brasil. Rev Bras Saude Mater Infant. 2009;9:157-165. DOI: 10.1590/S151938292009000200005.

11. Scholte M, Calsbeek H, Sanden MWGN, et al. Quality of physical therapy from a patient's perspective; factor analysis on web-based survey data revealed three dimensions on patient experiences with physical therapy. BMC Health Services Research. 2014;14:266.DOI 10.1186/1472-6963-14-266

12. Mendonça KMPP, Guerra RO. Desenvolvimento e validação de um instrumento de medida da satisfação do paciente com a fisioterapia. Rev Bras Fisioter. 2007;11(5):369-376. DOI: 10.1590/S141335552007000500007 .

13. Brasil. Comissão Nacional sobre Determinantes Sociais da Saúde. As causas sociais das iniquidades em saúde no Brasil. Brasília, DF; 2008. Extraído de[http://bvsms.saude. gov.br/bvs/publicacoes/causas_sociais_iniquidades.pdf], acesso em [13 de junho de 2017].

14. Gonçalves JR, Veras FEL, Matos ACM, et al. Avaliação da satisfação dos pacientes submetidos à intervenção fisioterapêutica no município de Campo Maior, PI. Fisioter Mov. 2011;24(1):47-56.DOI: 10.1590/S0103 51502011000100006.

15. Brito AT, Jesus CS, Fernandes MH. Fatores associados à satisfação dos usuários em serviços de fisioterapia. Rev Baiana de Saúde Pública. 2012;36(2):514-526. DOI: 10.22278/2318-2660.2012.v36.n2.a473.

16. Lopes JL, Cardoso MLAP, Alves VLS, et al. Satisfação de clientes sobre cuidados de enfermagem no contexto hospitalar. Acta Paul Enferm. 2009;22(2):136-41. DOI: $10.1590 /$ S0103-21002009000200004.

\section{Como citar este artigo:}

Camargo A, Gonçalves JG, Mazzo DM, Schleder JC. Avaliação da satisfação do atendimento fisioterapêutico em ambiente hospitalar. .Rev. Aten. Saúde. 2019;17(60): 19-27. 